\title{
Frontières
}

\section{Illustration de la résilience}

\section{Bernard Lefebvre}

Volume 22, numéro 1-2, automne-printemps 2009-2010

Résilience et deuil

URI : https://id.erudit.org/iderudit/045036ar

DOI : https://doi.org/10.7202/045036ar

Aller au sommaire du numéro

Éditeur(s)

Université du Québec à Montréal

ISSN

1180-3479 (imprimé)

1916-0976 (numérique)

Découvrir la revue

Citer cet article

Lefebvre, B. (2009). Illustration de la résilience. Frontières, 22(1-2), 120-122.

https://doi.org/10.7202/045036ar

Ce document est protégé par la loi sur le droit d'auteur. L'utilisation des services d'Érudit (y compris la reproduction) est assujettie à sa politique d'utilisation que vous pouvez consulter en ligne.

https://apropos.erudit.org/fr/usagers/politique-dutilisation/
Cet article est diffusé et préservé par Érudit.

Érudit est un consortium interuniversitaire sans but lucratif composé de l’Université de Montréal, l'Université Laval et l'Université du Québec à Montréal. Il a pour mission la promotion et la valorisation de la recherche. https://www.erudit.org/fr/ 


\section{ILLUSTRATION DE LA RÉSILIENCE}

\section{Bernard Lefebvre \\ Professeur retraité du Département des sciences de l'éducation, UQAM}

N'étant pas spécialiste en la matière, je me réfère au dictionnaire Robert pour saisir la signification du mot résilience. En physique, il se rapporte à la résistance des matériaux à un choc. Transposé dans le domaine psychosocial, c'est l'attitude d'une personne ayant la capacité de réagir positivement aux chocs qu'elle reçoit dans sa vie, que ce soit un deuil, un accident, une perte d'emploi ou d'argent, etc.

Dans notre vie, nous avons tous rencontré des hommes et des femmes qui ont subi de durs coups et qui ont su non seulement y résister, mais trouver des solutions pour répondre aux exigences de chaque situation et aux responsabilités qui leur incombaient.

Voici l'histoire de quatre personnes qui illustrent ce qu'est la résilience en action. Émile, Madame Lépine, Pierrette et Juliette, qui ont suscité mon admiration par leur courage dans l'épreuve et leur façon personnelle de réagir. Malgré leur souffrance, ces personnes ont réussi à relever positivement les défis que la vie leur a imposés. Nous verrons comment elles ont été résilientes.

\section{ÉMILE, UN RÉSILIENT}

Mon père Émile fut un résilient avant l'apparition de ce terme. Il naquit en 1884 , à Oka. À l'âge de quatre ans, alors que la dernière voiture quittait la cour derrière la maison de ses parents, le cheval partit brusquement. La voiture bouscula l'enfant qui venait à peine de sortir et une roue lui passa sur une jambe. Il fut traité selon la médecine de l'époque. Une plaie se forma au niveau de la hanche et ne guérit pas. Dire qu'aujourd'hui ce serait probablement une banale infection maîtrisée par les antibiotiques. La plaie arrêta de couler et se referma. Mais un autre problème surgit: la tête du tibia se coinça dans la hanche et l'articulation ne fonctionna plus, de telle sorte qu'Émile ne put redresser sa jambe qui devint plus courte que l'autre. Il était devenu infirme de la jambe droite, se déplaçait maintenant difficilement avec l'aide d'une canne et devait porter une bottine orthopédique.

J'imagine l'adolescent qui prend conscience de sa silhouette différente de celle des autres et de l'impossibilité de pratiquer les sports de son âge, qui se demande comment il pourra gagner sa vie. Pourra-t-il, comme les autres, rêver de se marier et de fonder une famille?

Il finit par choisir le métier de couturier et de tailleur d'habits pour homme. Il s'engagea comme apprenti et commença au bas de l'échelle, c'est-à-dire en livrant à pied ou en tramway les vêtements commandés par les clients, et cela sans salaire. Dans ses temps libres, il observait comment travaillaient les tailleurs d'expérience. L'un d'eux remarqua le jeune et lui proposa de lui montrer la coupe après les heures de travail, car il faut faire sur mesure le patron pour chaque client, la confection en série n'existant pas encore. C'est ainsi qu'à dix-huit ans, il s'est confectionné un habit sur mesure en tenant compte des modifications à faire à son pantalon vu sa difformité. Après quelques années de pratique, il acquit sa propre boutique et ne travailla plus pour le compte d'autrui.

De santé fragile, il craignait l'avenir, se demandant si la maladie ne le rattraperait pas. Il se prémunit contre ces peurs en faisant des épargnes et en administrant ses revenus avec sagesse. Il se demanda s'il pouvait avoir des enfants en santé et sans infirmité. À trente-six ans, il proposa le mariage à Antoinette Coutlée, une amie d'enfance de trois ans sa cadette. Elle lui rappela qu'elle lui avait dit, lorsqu'elle avait douze ans, qu'il ne marierait personne d'autre qu'elle. Ils eurent trois enfants et je suis le benjamin.

Il passa à travers la crise économique de 1929 à 1939. Les clients, sans travail, arrêtèrent d'encourager le tailleur. Les plus fidèles fréquentaient encore sa boutique comme lieu de réunion pour jouer aux dames. Il dut fermer sa boutique pour réduire ses dépenses. Ayant acquis quelques propriétés, il avait des locataires qui ne payaient plus leur loyer et qui vivaient de l'aide gouvernementale 
appelée secours direct. Mon père percevait pour chaque locataire une partie du faible loyer. Plusieurs n'ont jamais comblé les quelques dollars de différence. Avec ces maigres revenus, mon père devait faire vivre sa famille, finir de payer ses propriétés, les réparer si nécessaire et payer les taxes foncières.

Émile eut une tumeur au cervelet à l'automne 1951, perdit conscience et décéda dans les jours qui suivirent. Lui qui appréhendait de ne plus pouvoir marcher dans sa vieillesse, ce malheur ne lui arriva pas; il fut plutôt terrassé par une maladie mortelle. J'avais alors 21 ans et je commençais à peine à cheminer dans la vie.

Mon père dut supporter son infirmité, avec les souffrances et les incapacités inhérentes à sa situation. Il n'a pas apprécié son image corporelle et n'a pas vécu une jeunesse insouciante. Il a dû prendre sa vie en main. Il avait de la volonté. Il a fait fructifier ses capacités et il a développé ses habiletés. Il s'est instruit par lui-même, ce qu'on appelle maintenant être autodidacte. De plus, le métier qu'il a choisi était adapté à sa condition. Non sans difficulté, il a accepté sa situation et s'en est accommodé.

Mon père a vécu son enfance et son adolescence dans une famille stable et aimante, composée de deux sœurs et de deux frères. Il a lui-même fondé une famille stable. Ses croyances religieuses l'ont soutenu dans ses épreuves, lui ont donné du courage dans l'adversité. S'il a été bien entouré de parents et de quelques amis, il croyait en l'existence d'autres personnes habitant un monde supérieur qu'il pouvait invoquer et dont il espérait recevoir aide et assistance. Il vivait dans la confiance et l'espérance.

Mon père Émile a reçu un dur coup dès son enfance. Il en a gardé les stigmates durant toute sa vie. Plutôt que de se laisser abattre et de s'avouer vaincu, il a résisté sans se laisser écraser par la maladie et par son infirmité. Entouré des siens et à l'aide de ses valeurs morales et religieuses, il a résisté au choc et il fut donc résilient avant l'apparition du terme. Émile fut un homme valeureux comme beaucoup d'autres à son époque et comme on en rencontre fréquemment aujourd'hui. C'est la preuve qu'il est toujours possible de rebondir à condition de s'aider soi-même.

Cette histoire vécue ne tient pas du roman. La vie n'a pas fait de cadeau à mon père. Émile illustre bien ce qu'est la résilience.

\section{MADAME LÉPINE, LA VAILLANCE INCARNÉE}

Je présente brièvement cette dame Lépine, une amie de la famille, que j'ai connue dans mon enfance et qui aurait eu l'âge d'être ma grand-mère. Son mari travaillait pour la voirie, à la Ville de Montréal. De ce couple naquirent deux filles. Madame arrondissait le revenu familial en cousant chez elle pour des tailleurs d'habits pour homme. Tout allait bien jusqu'au jour où son mari fut terrassé par une crise cardiaque. Rappelons que vers 1920, la médecine n'avait pas fait tous les progrès que nous lui connaissons aujourd'hui.

La famille était amputée de son chef et la mère est restée seule avec deux fillettes en bas âge. Les services sociaux n'existaient pas. L'œuvre de la Société Saint-Vincent de Paul offrait un dépannage temporaire et sporadique selon les fonds dont elle disposait et ne pouvait compter que sur la générosité des paroissiens. Les gens ne s'y référaient qu'en cas de nécessité extrême. De façon générale, la plupart considéraient comme une honte de devoir demander la charité. Alors, madame Lépine rassembla tout son courage et après les funérailles et la mise en terre de son mari, elle passa outre à ses émotions et à son découragement. Comme des commandes de couture étaient restées en attente, l'après-midi même, elle se remit à sa machine à coudre. Sa famille dépendait désormais d'elle pour sa subsistance.

Ses enfants grandirent. Ses filles se marièrent. Madame Lépine demeura chez l'une d'elles après avoir cessé de travailler et vécut une heureuse vieillesse entourée de sa famille.

Elle resta toujours connectée à la vie actuelle en étant en relation avec des gens de divers âges, adultes et adolescents. Elle lisait quotidiennement le journal, allait de temps à autre au cinéma et fréquentait quelques amis. Madame Lépine était une femme vivante, active et aimable.

Malgré son deuil et son travail exigeant, elle avait réussi à faire vivre décemment sa famille en aimant ses enfants. Un dimanche midi d'hiver, elle revint de la messe, entra chez elle, s'assit... et son cœur arrêta de battre. Elle avait bien accompli sa mission en étant résiliente.

\section{PIERRETTE, LA DÉLAISSÉE RÉSILIENTE}

Pierrette, mariée à Maurice, fut pour nous une grande amie. Ce couple eut trois fils. Tout semblait bien aller pendant de nombreuses années. Monsieur possédait une entreprise qui était florissante, mais qui l'obligeait souvent à entrer chez lui assez tard le soir. Les enfants grandissaient et poursuivaient leurs études. Le mari se désintéressa progressivement de sa famille. Son humeur changea. Il se mit à fréquenter des clubs où il passait en partie ses soirées. Soudain, il se découvrit le goût de voyager fréquemment en Amérique du Sud. Un jour, il annonça à sa femme qu'il divorçait et qu'elle devait quitter la maison. Pierrette dit au juge que Maurice devait lui payer une pension alimentaire. Elle se fit répondre qu'elle n'avait qu'à aller travailler, mais elle avait déjà soixante ans.

Femme à la maison, elle avait élevé sa famille et vu aux besoins de son mari. Elle se fit mettre à la porte et elle a été réduite à la pauvreté. Monsieur s'était organisé pour faire émigrer une femme divorcée avec ses trois enfants. Pierrette n'a pas eu de chance. Elle a subi une dure épreuve, mais sa spiritualité est demeurée intacte et l'a sauvée. Ensuite, elle a travaillé pour diverses œuvres caritatives sans que son travail ni son dévouement ne soient reconnus. Quand ses services n'étaient plus requis, on la remerciait sans se préoccuper de sa subsistance future.

Heureusement pour elle, elle alla rejoindre l'un de ses fils qui administrait une maison qui hébergeait des jeunes gens en difficulté. Elle passa quelques années à effectuer diverses tâches afin de prodiguer des soins attentifs à des jeunes déshérités. Pierrette ne fut pas chanceuse, mais elle s'est ressaisie et a poursuivi courageusement son chemin. Comme bien d'autres, cette discrète personne n'a pas fait de publicité ou de tapage. Elle a été tout simplement résiliente.

\section{JULIETTE OU LE DÉVOUEMENT}

Juliette quitta la campagne et déménagea à Montréal avec toute sa famille. Plus tard, elle se maria à Louis. Ils eurent six enfants mais un seul a survécu. Sa sœur cadette, Valéda, vint la rejoindre et demeura chez elle. Un jour, elle rencontra un bel Irlandais et se maria. Comme il était courant à cette époque de la décennie 1920, le jeune couple aménagea avec Juliette et Louis. Il était tout naturel que ce nouveau couple eût des enfants; il donna naissance à trois filles nommées Thérèse, Henriette et Pauline. La famille filait le parfait bonheur. La cadette n'était pas encore née lorsque Thomas, le futur père, tomba malade et dut s'aliter. On diagnostiqua un problème cardiaque. Prenant du repos, étendu sur son lit, il lisait un article de Henri Bourassa dans le journal Le Devoir puis demanda un verre d'eau. Quand Valéda, son épouse, le lui apporta, il venait de décéder d'une crise cardiaque.

Juliette et Louis aidèrent Valéda dans ce moment difficile. Plus tard, cette dernière se trouva un emploi et Juliette prit soin des petites filles. Quand elles atteignirent respectivement 6 ans, 4 ans et 3 ans, Valéda dut entrer à l'hôpital pour une intervention chirurgicale. Au cours des jours suivants, elle contracta une pneumonie. Il 
faut savoir qu'autour de 1930, la pénicilline n'était pas encore couramment utilisée et qu'une telle maladie était souvent funeste. Dans la mi-vingtaine, la maladie l'emporta laissant les trois fillettes orphelines de père et de mère. Un conseil de famille accepta que Juliette et Louis aient la garde légale des enfants sans qu'ils exigent aucune rétribution monétaire. Ils reprenaient leur rôle de parents à part entière même si leur fils avait atteint l'âge de 16 ans. Pour les enfants, c'était le mieux qui pouvait leur arriver, car elles demeuraient ensemble dans le milieu où elles vivaient déjà. Ils appelaient Juliette aussi bien ma tante que maman.

Louis accepta de se charger des dépenses supplémentaires qu'exigeait sa famille agrandie. La besogne de la mâ̂tresse de maison augmentait. Ce couple a relevé le défi, l'État ne jouant pas le rôle de suppléance qu'on lui connaît aujourd'hui. Juliette et Louis, qui n'étaient plus dans leur prime jeunesse, n'ont pas pensé à ce qu'on appelle aujourd'hui leur qualité de vie. Ils ont pris soin de leurs nièces à qui ils ont dispensé temps, argent et amour comme si elles étaient leurs propres enfants.
Les trois fillettes ont grandi et ont poursuivi des études. Thérèse, l'aînée, entra chez les religieuses de la Providence, la deuxième, Henriette, est devenue mon épouse, et la cadette, Pauline, a gardé Juliette chez elle quand elle s'est mariée. Malade et alitée, Juliette se leva une dernière fois pour parler au téléphone à sa fille qui lui annonçait la naissance de son enfant, qui s'appellerait Juliette. L'état de la grand-maman continua de se dégrader et elle s'éteignit au cours de la soirée. Pour elle, c'était mission accomplie. Henriette et son mari gardent un souvenir attendri de cette tante, mère et belle-mère, qui ne faisait pas de bruit, mais qui était industrieuse et efficace. Toute sa vie, elle a pris soin des gens qui l'entouraient et elle s'est dépensée pour eux. Elle l'a fait par amour et non par sacrifice. Elle était une vraie adulte et agissait comme telle. Son réseau de parents et d'amis était suffisant pour avoir une vie sociale agréable et gratifiante.

$$
\text { *** }
$$

Cette galerie de portraits illustre bien la résilience. Chacun l'est à sa manière. Il ne s'agit pas de la découverte du siècle. C'est là une attitude et un comportement psy- chosocial qui sont propres à l'être humain raisonnable qui subit des contrecoups de toutes sortes et qui réagit, à la suite de la prise de conscience de ce qui lui arrive, qui souffre de la situation émotivement ou physiquement, après un décès, un accident ou lorsqu'il est frappé par une maladie. Ne constatons-nous pas que des malheurs semblables sont arrivés dans les familles de notre entourage. Cela fait partie de la commune humanité. Nous n'avons d'autres choix que d'assumer la réalité de ce qui nous arrive et de trouver les moyens de vivre malgré l'épreuve.

Se refermer sur soi-même et couper ses relations avec les autres personnes ne peuvent conduire qu'à la dépression. C'est dans ces phases de découragement que se produisent des suicides et des divorces, sans compter les disputes et les rancunes parce que, dans un tel état émotionnel, il est plus facile de rejeter la responsabilité sur les autres.

Nous connaissons de réputation une championne olympique, un médecin ou un avocat handicapés. Ils ont exploité leurs capacités demeurées intactes. Tous nous possédons de nombreuses ressources à faire fructifier pour réinventer l'avenir. 